\title{
A160 IFN-GAMMA PROMOTES FIBROBLAST-LIKE SYNOVIOCYTES MOTILITY
}

T Karonitsch, K Dalwigk, R Byrne, B Niedereiter, E Cetin, A Wanivenhaus, C Scheinecker, J S Smolen, H P Kiener Medical University of Vienna, Vienna, Austria

\subsection{6/ard.2010.129650k}

Rheumatoid arthritis (RA) is the most common chronic inflammatory joint disease. In RA, a systemic autoimmune response translates into an inflammatory attack on the synovium that yields the formation of an aggressive cell mass called pannus which attaches to, encroaches over and destroys the articular cartilage. Cartilage destruction is mediated by fibroblast-like synoviocytes (FLS) which are the prevailing cell type of the aberrant pannus tissue. Here we demonstrate that interferon gamma (IFN-gamma), which is abundantly expressed in rheumatoid synovitis, promotes FLS motility through a signalling cascade that involves the activation of focal adhesion kinase (FAK).

To investigate a role for IFN-gamma in facilitating pannus formation and FLS motility in arthritis, we performed migration assays using modified Boyden chambers. Strikingly, IFNgamma-stimulated FLS demonstrate an increased migratory activity compared with unstimulated FLS. Using immunofluorescence and confocal microscopy, we further analysed the FLS cellular response to IFN-gamma. Cell spreading assays revealed prominent membrane protrusions and multiple focal adhesions connected to longitudinal actin stress fibres after $15 \mathrm{~min}$ in culture for FLS that were exposed to IFN-gamma. By contrast, unstimulated FLS rarely formed membrane protrusions and few focal contacts were enmeshed in cortical actin. Thus, IFN-gamma stimulation had a profound impact on FLS actin cytoskeletal reorganisation and focal adhesion development. Since actin reorganisation, cell-to-matrix adhesion and cell motility are partly controlled by FAK, we hypothesised that IFN-gamma modulates FAK activity. Indeed, western blot analysis using a monoclonal antibody to Tyr397-phosphorylated FAK revealed that IFN-gamma stimulation results in FAK activation. In Stat1-deficient U3A cells, IFN-gamma stimulation still resulted in FAK phosphorylation. By contrast, inhibition of JAK efficiently abrogated IFN-gamma-induced activation of FAK. Thus, the activation of FAK by IFN-gamma was dependent on JAK but not Stat1.

These studies suggest a role for IFN-gamma in the mesenchymal tissue response to inflammation and may provide insight into FLS behaviour and function in arthritis, especially rheumatoid arthritis. 